\title{
Evidence that CED-9/Bcl2 and CED-4/Apaf-1 localization is not consistent with the current model for $C$. elegans apoptosis induction
}

\author{
E Pourkarimi ${ }^{1}, \mathrm{~S}$ Greiss $^{1,2}$ and A Gartner ${ }^{*, 1}$
}

In C. elegans, the BH3-only domain protein EGL-1, the Apaf-1 homolog CED-4 and the CED-3 caspase are required for apoptosis induction, whereas the Bcl-2 homolog CED-9 prevents apoptosis. Mammalian B-cell lymphoma 2 (Bcl-2) inhibits apoptosis by preventing the release of the Apaf-1 (apoptotic protease-activating factor 1) activator cytochrome $c$ from mitochondria. In contrast, $C$. elegans CED-9 is thought to inhibit CED-4 by sequestering it at the outer mitochondrial membrane by direct binding. We show that CED-9 associates with the outer mitochondrial membrane within distinct foci that do not overlap with CED-4, which is predominantly perinuclear and does not localize to mitochondria. CED-4 further accumulates in the perinuclear space in response to proapoptotic stimuli such as ionizing radiation. This increased accumulation depends on EGL-1 and is abrogated in ced-9 gain-of-function mutants. CED-4 accumulation is not sufficient to trigger apoptosis execution, even though it may prime cells for apoptosis. Our results suggest that the cell death protection conferred by CED-9 cannot be solely explained by a direct interaction with CED-4.

Cell Death and Differentiation (2012) 19, 406-415; doi:10.1038/cdd.2011.104; published online 2 September 2011

Seminal studies on $C$. elegans apoptosis that occurs during the invariant development of the worm are important for our general understanding of the mechanism of apoptosis induction. EGL-1, CED-4 and CED-3 are conserved proteins required for the vast majority of the 131 cell deaths that occur during nematode development. ${ }^{1-3}$ The sole nematode B-cell lymphoma 2 (Bcl-2) family member CED-9 (cell death abnormal 9) is required to protect healthy cells from undergoing apoptosis. ${ }^{3}$ The ced-9 loss-of-function mutants undergo inappropriate apoptosis in cells that are normally destined to survive, whereas a ced- 9 gain-of-function mutant is defective for apoptosis induction in cells normally destined to die. ${ }^{3}$ In the worm, apoptosis generally involves the transcriptional upregulation of the $\mathrm{BH}$-only domain protein egg-laying defective 1 (EGL-1), mediated by a combination of transcriptional regulators acting on egl-1 in the specific cells destined to die. ${ }^{2,4}$ EGL-1 antagonizes CED-9 that, in turn, antagonizes the proapoptotic activity of CED-4, the apoptotic proteaseactivating factor 1 (Apaf-1) homolog. ${ }^{2,4}$ In contrast to mammalian systems where Apaf-1 activation is linked to the release of cytochrome $c$ from mitochondria, ${ }^{5}$ in $C$. elegans the decision between the survival or the death of a cell is reported to be largely regulated by the direct interaction of CED-4 and CED-9. ${ }^{6}$ Mitochondrial CED-9 directly binds proapoptotic CED-4 to inhibit apoptosis induction in cells destined to survive. Upon transcriptional induction, EGL-1 binding to CED-9 leads to a conformational change in CED-9, resulting in the release of CED-4 and its translocation to the nuclear periphery. ${ }^{6}$ This event leads to CED-4 oligomerization and the induction of apoptosis through CED-3 caspase activation. ${ }^{6-8}$ Recent structural evidence suggests that the activated CED-4 complex comprises a funnel-like octameric structure with fourfold symmetry, each unit being defined by an asymmetric CED-4 dimer. ${ }^{9}$ The cavity of this structure provides space for a CED-3 dimer, and the induced proximity of these two CED-3 molecules is likely to be required for caspase activation. ${ }^{9}$ The notion that EGL-1/CED-9 and CED-9/CED-4 can directly interact is supported by yeast two-hybrid analysis, by co-immunoprecipitation studies in heterologous systems and by crystallographic evidence showing that CED-9 can bind to a CED-4 dimer. ${ }^{8-12}$ Previous cytological studies in C. elegans embryos appeared to show the colocalization of CED-9 and CED-4 on mitochondria in nonapoptotic cells, and indicated that CED-4 translocation to the perinuclear space was linked to apoptosis induction. ${ }^{6}$ Although there is no evidence for cytochrome $c$ release in $C$. elegans, the release of other mitochondrial factors such as AIF and EndoG as well as mitochondrial fragmentation has been implicated in apoptosis induction. ${ }^{13,14}$ However, the apoptosis-defective phenotypes associated with the depletion of these factors are very weak and the importance of mitochondrial fragmentation has been debated. ${ }^{13,14}$ Furthermore, there is evidence that CED-9 permeabilizes membranes in vitro and the overexpression of CED-9 and Bcl-2 has been shown to lead to mitochondrial fusion in mammalian tissue culture and in C. elegans. ${ }^{15-17}$ Here we revisit the localization of CED-9 and

\footnotetext{
${ }^{1}$ Wellcome Trust Centre for Gene Regulation and Expression, University of Dundee, Dundee DD1 5EH, UK

*Corresponding author: A Gartner, Wellcome Trust Centre for Gene Regulation and Expression, University of Dundee, Down Street, Dundee, DD1 5EH, UK.

Tel: + 441382 385809; E-mail: a.gartner@dundee.ac.uk

${ }^{2}$ Current address: MRC Laboratory of Molecular Biology, Cambridge CB2 0QH, UK.

Keywords: CED-4; Apaf-1; CED-9; Bcl-2; C. elegans; apoptosis

Abbreviations: ced, cell death abnormal; egl, egg-laying defective; SI-OMX, structural illumination opticam experimental microscopy; Apaf-1, apoptotic proteaseactivating factor 1; Bcl-2, B-cell lymphoma 2; ER, endoplasmic reticulum

Received 10.12.10; revised 21.6.11; accepted 28.6.11; Edited by E Baehrecke; published online 02.9.11
} 
CED-4. We confirm that CED-9 is located at mitochondria and show that it forms distinct foci. Unexpectedly, CED-4 does not localize at mitochondria in the germ line and we provide strong evidence that this is also the case during somatic development. Using the $C$. elegans germ line as a model we show that CED-4 perinuclear accumulation might prime cells for apoptosis induction but is not sufficient to induce apoptosis.

\section{Results}

We previously generated two specific CED-4 antibodies, which we employed for immunostaining of dissected
C. elegans germ lines. ${ }^{18}$ We confirmed the perinuclear staining pattern we observed with a third independently generated CED-4 antibody, which specifically recognizes CED-4 (Supplementary Figures $1 \mathrm{a}$ and $\mathrm{b}$ ). ${ }^{18}$ Using highresolution imaging, we found that CED-4 is expressed in the entire germ line, located primarily around the nucleus with additional much weaker granular structures occurring in the cytoplasm (Figure 1a). As the current model of apoptosis regulation depends on the direct interaction between CED-4 and mitochondrial CED-9, we next tried to establish whether CED-4 staining was associated with mitochondria as previously reported. ${ }^{6}$ We visualized mitochondria by staining a

0 Gy

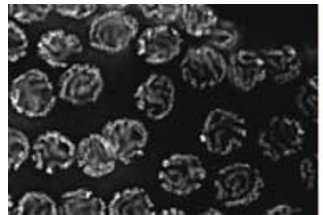

DAPI

b

120 Gy

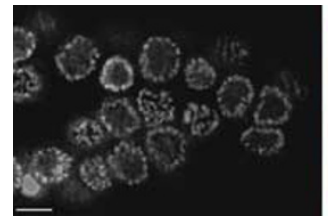

DAPI

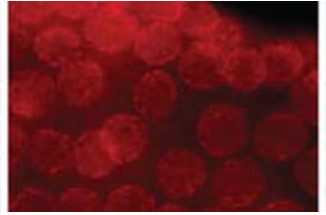

CED-4

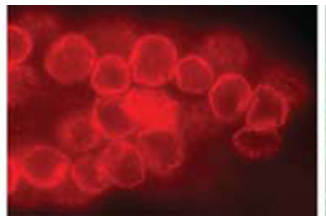

CED-4

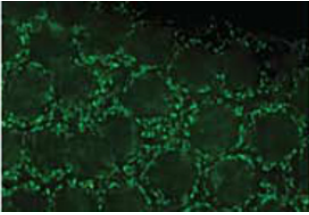

Mitochondria

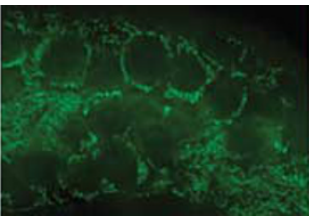

Mitochondria

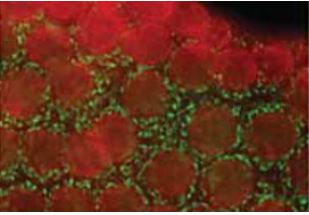

CED-4, Mitochondria

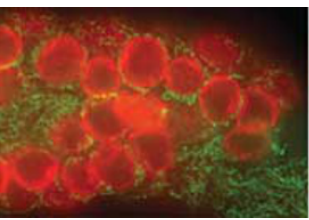

CED-4, Mitochondria

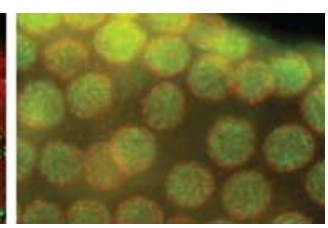

CED-4,SIR-2

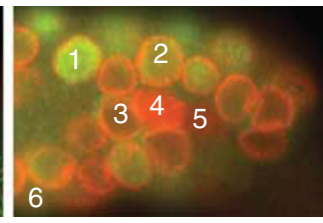

CED-4,SIR-2 c

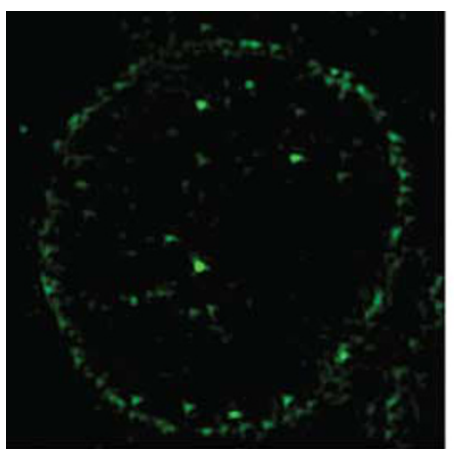

CED-4

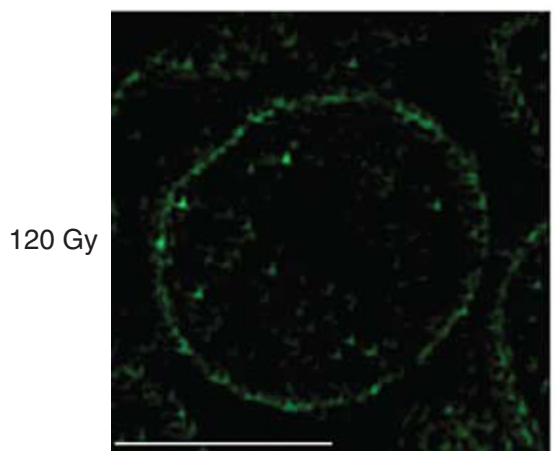

CED-4

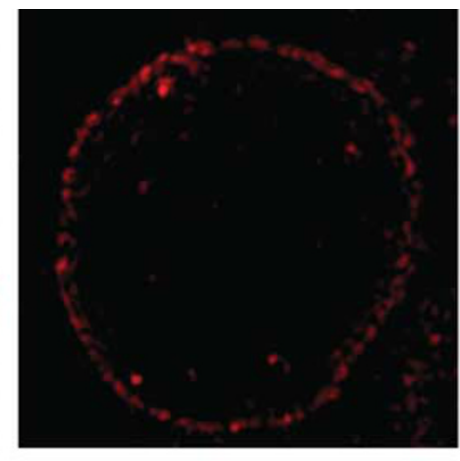

Nuclear pore complex

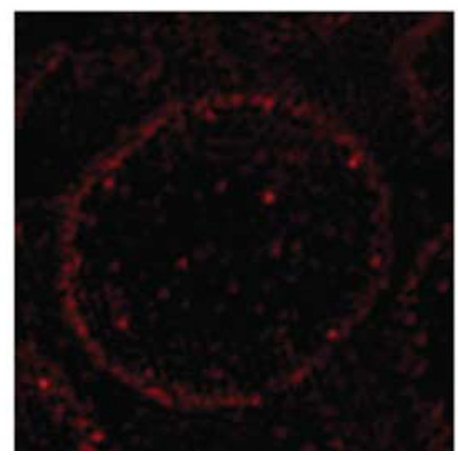

Nuclear pore complex
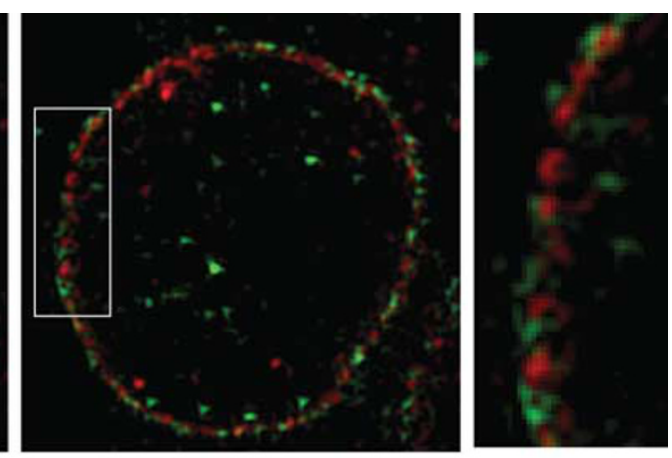

Merge
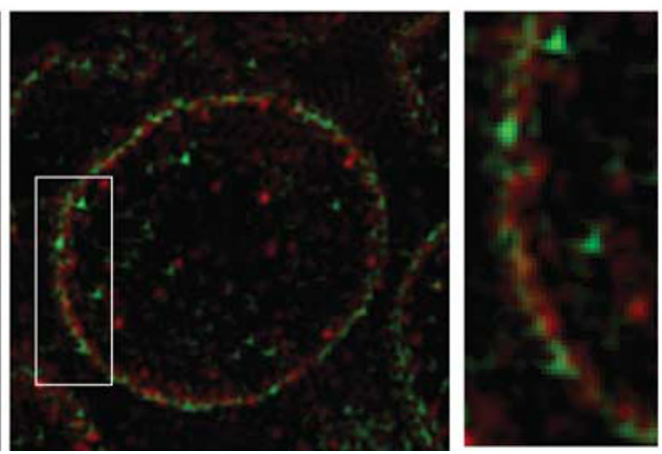

Merge

Figure 1 CED-4 is not localized on mitochondria in the germ line. (a) Representative pictures of late-stage pachytene germ cells were taken by Deltavision microscopy. CED-4 staining was done with the CED-4 9104.1 antibody. Mitochondria were stained with anti-ATP synthase and anti-cytochrome $c$ antibodies. The corresponding 3D stacks of the images are shown in Supplementary Movie 1 (CED-4) and Supplementary Movie 2 (CED-4/mitochondia); scale bar: $6 \mu$ m. (b) CED-4 perinuclear accumulation upon treatment with IR. A representative healthy cell, and corpses indicative of different stages of apoptosis progression are indicated in the right panel (1-6). A blow up of these cells is shown in Figure 6a. Note that the CED-4 signal intensity in (b) is lowered by $15 \%$ compared with (a) to allow for better visualization. (c) OMX microscopy of single germ cells before and after IR (CED-4 green, nuclear pore complex red). Scale bar: $4 \mu \mathrm{m}$ 
dissected germ lines with a cocktail of monoclonal antibodies recognizing conserved mitochondrial proteins. Specificity for mitochondria was confirmed by the perfect overlap of the staining patterns of the different antibodies in germ lines (Supplementary Figure 2a) as well as embryos (Supplementary Figure 2b). To our surprise, we found that CED-4 does not colocalize with mitochondria in germ cells (for late pachytene cells see Figure 1a). The separate localization of CED-4 and mitochondria is most apparent when scanning through $Z$ stacks of representative germ lines (Supplementary Movie 1, CED-4; Supplementary Movie 2, CED-4/mitochondria). We found the same nonmitochondrial staining patterns for CED-4 in mitotic, transition zone, and both early- and late-stage pachytene germ cells (data not shown). We confirmed the absence of CED-4 mitochondrial staining with an additional CED-4 antibody we previously generated (Supplementary Figure 1b). Finally, we transformed worms with a construct encoding for CED-4::GFP driven by the ced-4 promoter. The fusion protein was functional, rescuing the apoptosis defect of the ced-4(n1162) loss-of-function allele (Figure 2a). When we analyzed CED-4 localization in the transgenic worms using immunofluorescence with anti-GFP antibodies, we again did not see any obvious colocalization of CED-4::GFP with mitochondria (Figure $2 \mathrm{~b}$ ). The same predominantly perinuclear CED-4 localization pattern was observed following detection of the CED-4::GFP fusion protein by direct fluorescence, a finding that excludes the possibility that our staining procedures led to a loss of mitochondrial CED-4 (Figure 2c). In summary, our data show that CED-4 does not detectably localize to mitochondria in the germ line. Similarly, labeling the endoplasmic reticulum (ER) did not reveal any colocalization of the weak granular cytoplasmic CED-4 signal with the ER (Supplementary Figure 3c). As previous studies reporting a direct interaction of CED-9 and CED-4 at mitochondria were performed in embryos, we reasoned that our results could be explained by the existence of a fundamental difference between the regulation of developmental apoptosis and germ cell apoptosis. We therefore wished to reassess the cellular localization of CED-4 and CED-9 during embryogenesis and larval development. Using our panel of CED-4 antibodies we never saw CED-4 localized at mitochondria. In contrast to our germ line stainings, we

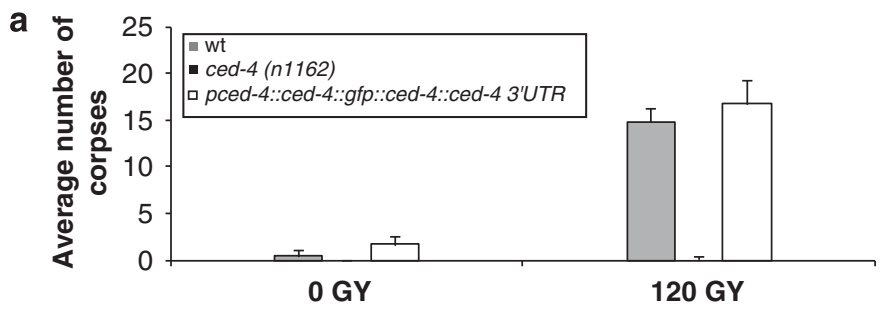

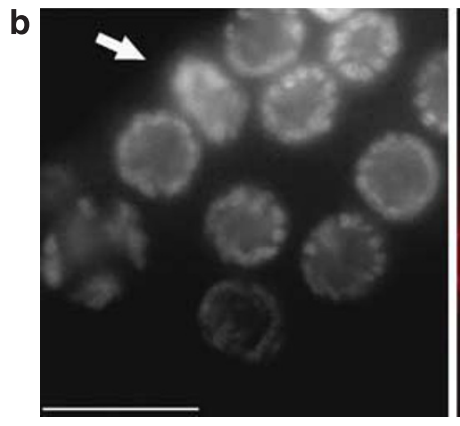

DAPI

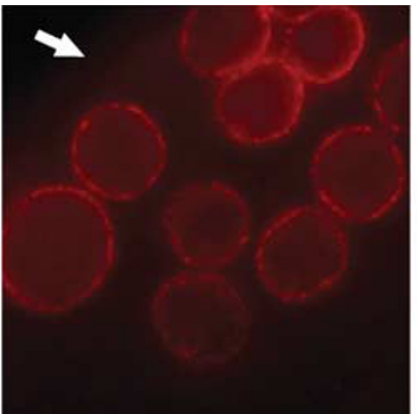

CED-4::GFP

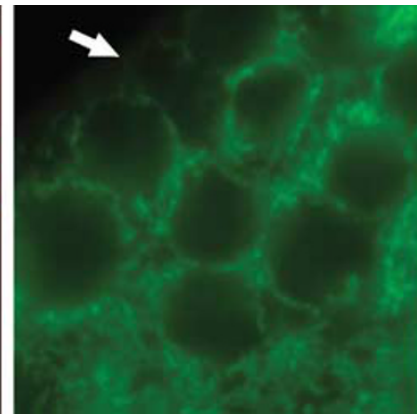

Mitochondria

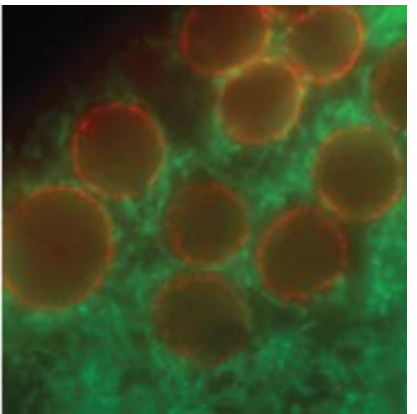

Merge

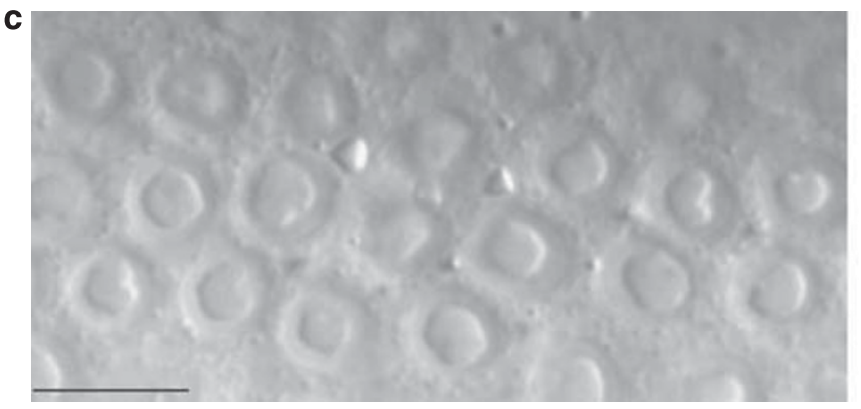

$\mathrm{DIC}$

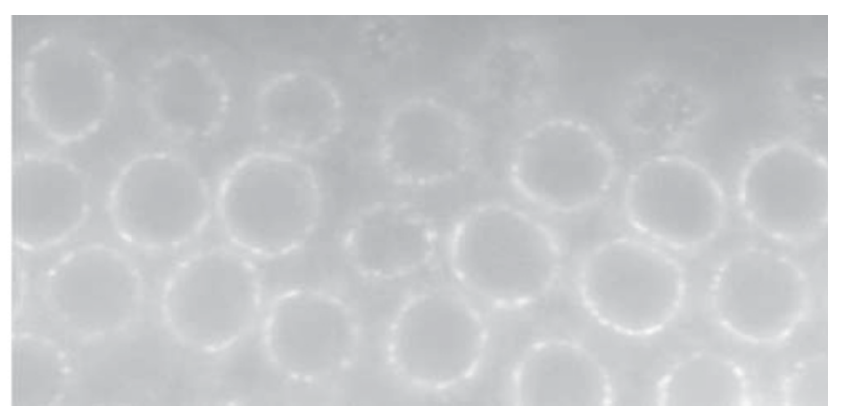

CED-4::GFP

Figure 2 Perinuclear localization of CED-4::GFP. (a) The ced-4 gt1767 Ex[pced-4::ced-4::gfp:::ced-4 3'UTR, unc-119(+ )] construct rescues the ced-4(n1162) apoptosis defect. Only worms carrying the CED-4::GFP transgene were scored. (b) CED-4::GFP staining using anti-GFP antibodies. A white arrow indicates a very-late-stage corpse, where mitochondria are fragmented, chromatin is condensed and CED-4 staining is absent (see Figures $1 \mathrm{~b}$ and 6 ). (c) Direct visualization of CED-4::GFP using fluorescence microscopy. The left panel shows DIC image of pachytene-stage germ cells and the right panel overlaps with fluorescence image. Scale bar: $10 \mu \mathrm{m}$ 
could not confirm the presence of CED-4 at nuclei with absolute certainty, because of unspecific perinuclear staining that was not present in germ lines (data not shown). We therefore analyzed the same functional CED-4::GFP lines we had used for assessing the localization of CED-4 in the germ line. In agreement with our germ cell data, we found no embryonic cells where CED-4::GFP staining colocalized with mitochondria, and in all cells where CED-4 was detectable it localized around the nucleus (Figure 3; Supplementary Movie 3 , CED-4/mitochondria). We found the same perinuclear CED-4::GFP localization by directly detecting CED-4 by GFP fluorescence in embryos, larvae and adult worms (data not shown). Using antibodies kindly supplied by Barbara Conradt (Supplementary Figure 3a), we could confirm a partial overlap between CED-9 and mitochondria (Figure 3; Supplementary Movie 4, CED-9/mitochondria; Supplementary Movie 5, CED-4/CED-9, Supplementary Figure 3b); however, we were unable to detect any overlap between CED-4 and CED-9 (Figure 3, Supplementary Movie 5, CED-4/CED-9). Imaging CED-9 in early-stage embryos using deconvolution microscopy, we found that CED-9 staining was not evenly distributed along mitochondria but appeared somewhat punctate, often associated with but not perfectly overlapping with mitochondria (Supplementary Figure $3 \mathrm{~b}$ ). A punctate pattern was also observed in the germ line using the CED-9 antibody provided by the Conradt lab and the originally described CED-9 antibody (data not shown). ${ }^{6}$ To address CED-9 localization more precisely, we employed structural illumination opticam experimental microscopy(SI-OMX), a technique that allows an approximately twofold higher resolution compared with conventional deconvolution microscopy. ${ }^{19}$ Using OMX microscopy we observed a continuous staining of the inner mitochondrial membrane with anti-ATP synthase antibodies, whereas CED-9 staining appeared in distinct dots (Figure 4a). Zooming in on individual mitochondrial branches revealed that the CED- 9 dots tend to align next to the mitochondrial inner membrane (Figure 4b, Supplementary Movie 6). This lack of overlap is consistent with $\mathrm{OMX}$ microscopy resolving the bulk of mitochondrial cristae that define the inner mitochondrial membrane, identified by marker proteins, from CED-9 associated with the outer mitochondrial membrane. The localization of CED-9 foci next to the mitochondrial surface became most obvious following a 3D reconstruction of mitochondrial and CED-9 staining (Supplementary Movie 7). The CED-9 staining pattern that we observed is similar to the reported staining pattern of $\mathrm{Bcl}-2$ in mammalian cells. ${ }^{20,21}$ Unfortunately, because of technical limitations it was not possible to triple stain nematode tissue with CED-4, CED-9 and mitochondria-specific antibodies for analysis using the OMX microscope. In summary, our results suggest that there is no fundamental difference in CED-4 localization between hermaphrodite germ cells and somatic cells, and that CED-4 localization does not overlap with mitochondria and CED-9 in the vast majority of cell types during somatic development and in the germ line. To independently confirm the localization of CED-4 and CED-9, we developed procedures to achieve subcellular fractionation (see Materials and Methods). Using this procedure, and employing GAPDH as a cytosolic marker, a component of the mitochondrial ATP synthase as a mitochondrial marker and histone $\mathrm{H} 2 \mathrm{~A}$ as a nuclear marker, we confirmed enrichment of the respective compartments (Figure $4 \mathrm{c}$ ). Staining with CED-9 antibodies confirmed enrichment in the membranous fraction, whereas CED-4::GFP is enriched in the nuclear fraction (Figure 4c). Analogous results were obtained in independent experiments using CED-4 antibodies (data not shown). In addition, we could not find a direct interaction between CED-4 and CED-9 in co-immunoprecipitation experiments and upon mass spectroscopy of CED-4 precipitates (data not shown). Intriguingly, we found that CED-4 appears to colocalize with mitochondria and CED-9 in secondary spermatocytes and spermatids, cell types where apoptosis is not reported to occur ${ }^{22}$ and where a role for CED-4 and CED-9 cell death proteins is not known (Figure 5). These cells make out only a tiny fraction of a total worm extract. This CED-4 localization in spermatocytes and spermatids was observed with two independent CED-4 antibodies and by staining for CED-4::GFP (data not shown). In summary, our combined data suggest that CED-4 and CED-9 do not localize in the vast majority of cells.

We next wished to determine whether changes in CED-4 subcellular localization occur during apoptosis progression. For this, we focused our observations on the adult $C$. elegans germ line, as apoptosis can be readily induced by ionizing radiation in this tissue. ${ }^{23}$ We and others have reported that CED-4 accumulates at the nuclear periphery of germ cells in response to ionizing radiation. ${ }^{18,24}$ CED-4 accumulation occurs within $6 \mathrm{~h}$ of ionizing radiation and affects late pachytene as well as mitotic germ cells and transition zone nuclei (data not shown). ${ }^{24,25}$ The IR-dependent perinuclear accumulation of CED-4 appears strongest in late pachytene cells, which are the only germ cells competent to die by apoptosis (Figure 1b).

CED-4 perinuclear accumulation has previously been associated with apoptosis induction in ced-9 (If) embryos. ${ }^{6}$ To test if CED-4 accumulation correlates with apoptosis, we employed SIR-2.1 nuclear exclusion ${ }^{18}$ as early-stage, mitochondrial fragmentation as mid-stage and chromosome condensation as late-stage cytological markers for apoptosis progression. Using high-resolution imaging, we found that IRdependent CED-4 accumulation at the nuclear periphery does not directly correlate with apoptosis. Indeed, comparing individual cells that show IR-dependent CED-4 perinuclear accumulation (marked in the right panel of Figure $1 \mathrm{~b}$ ) revealed that some cells contained intact mitochondria, as well as SIR-2.1 localized to the nucleus (cell numbered (1), Figures $1 \mathrm{~b}$ and 6), whereas other cells were losing (2) or had lost nuclear SIR-2.1 (3) and contained fragmented mitochondria (3) and are thus corresponding to early- to mid-stage apoptotic cells (Figures $1 \mathrm{~b}$ and 6; CED-4; Supplementary Movie 8 CED-4/mitochondria; Supplementary Movie 9, CED-4/SIR-2.1). Thus, CED-4 perinuclear accumulation occurs in both healthy and apoptotic cells. It should also be noted that the dose of irradiation that we used only leads to a twofold reduction in egg laying, implying that a high proportion of germ cells can survive CED-4 perinuclear accumulation. ${ }^{23}$ Only late-stage corpses (4) showed evidence of chromatin fragmentation. These corpses also often showed excessive CED-4 staining (CED-4 hyperaccumulation) at the nuclear periphery and throughout the nucleus (4). In very-late-stage 


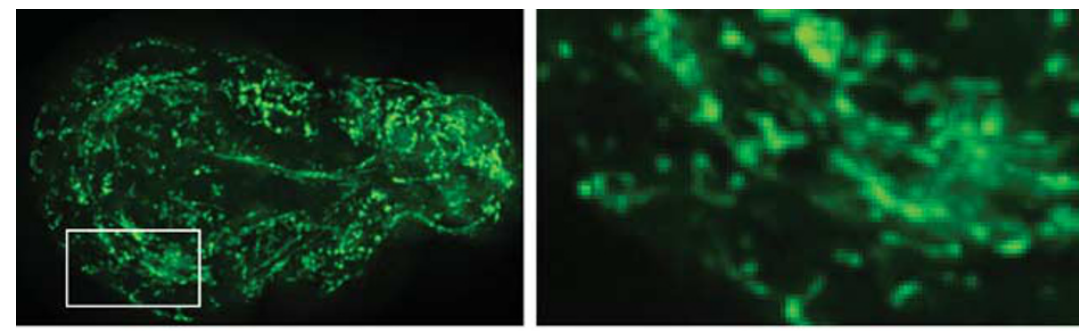

Mitochondria

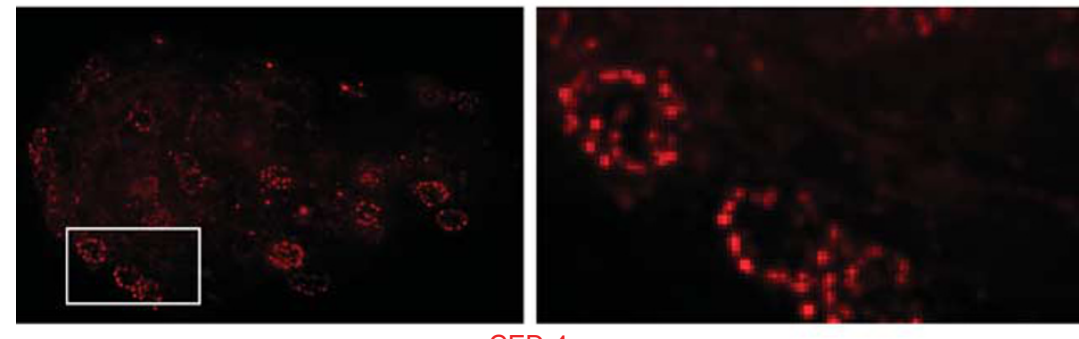

CED-4

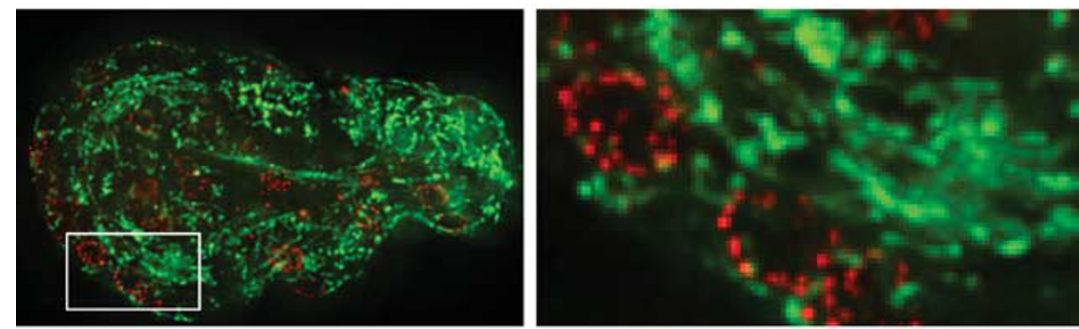

CED-4, Mitochondria

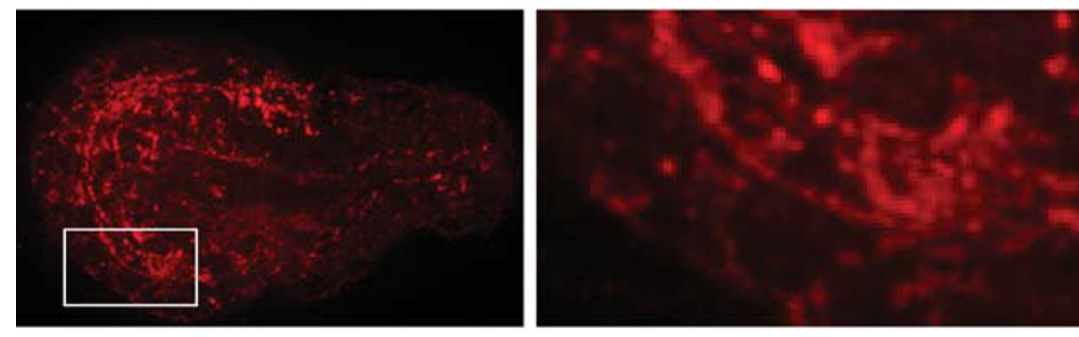

CED-9

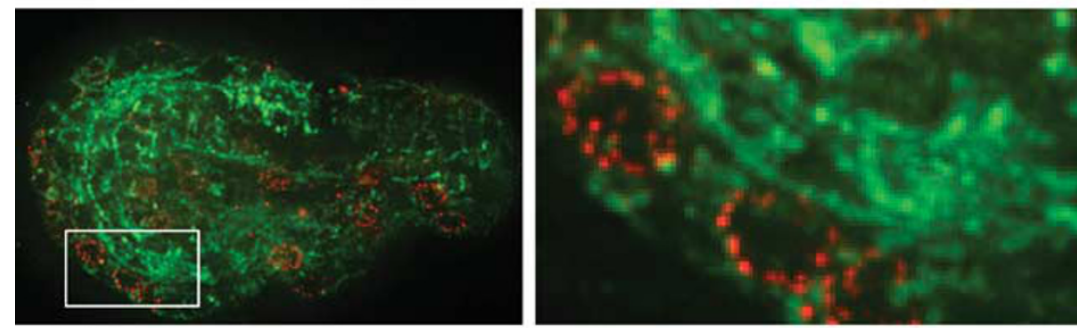

CED-4, CED-9

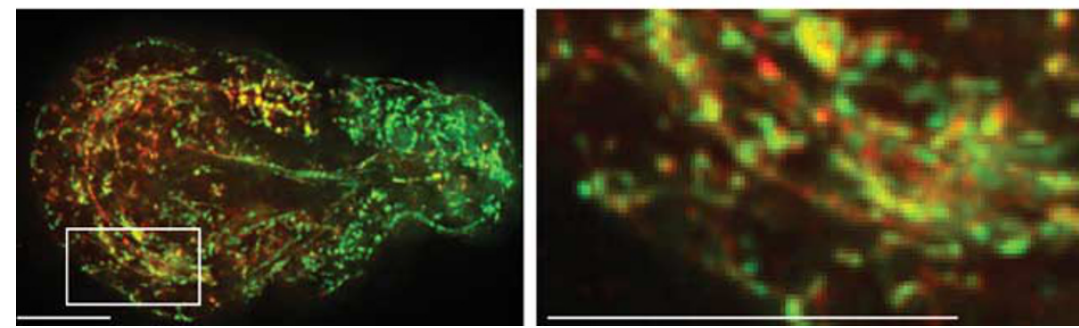

CED-9, Mitochondria

Figure 3 Staining of an embryo undergoing morphogenesis indicates that CED-4 does not colocalize with CED-9 and mitochondria. CED-4 is visualized using anti-GFP antibodies (see Materials and Methods). The image shown is a projection of six Z stacks. The 3D scans are shown in Supplementary Movies 3-5. Scale bar: 10 $\mu \mathrm{m}$ 


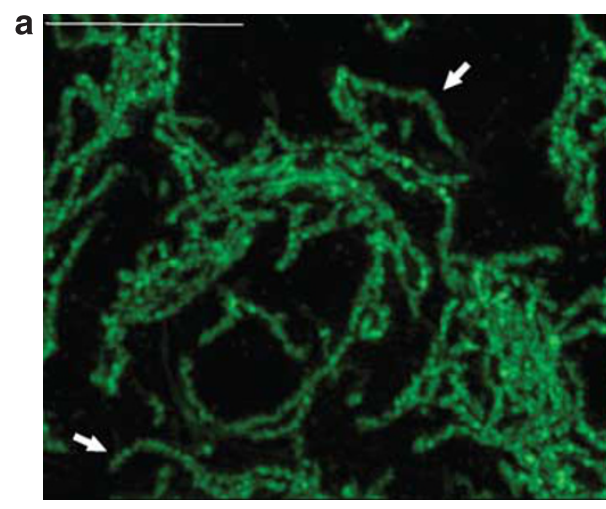

ATPsynthase

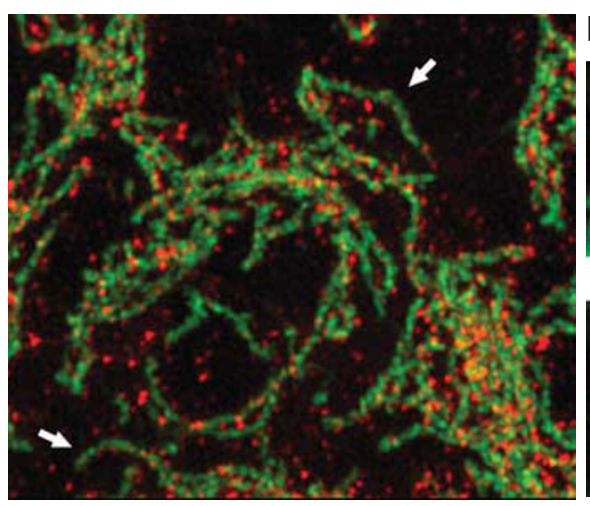

MERGE

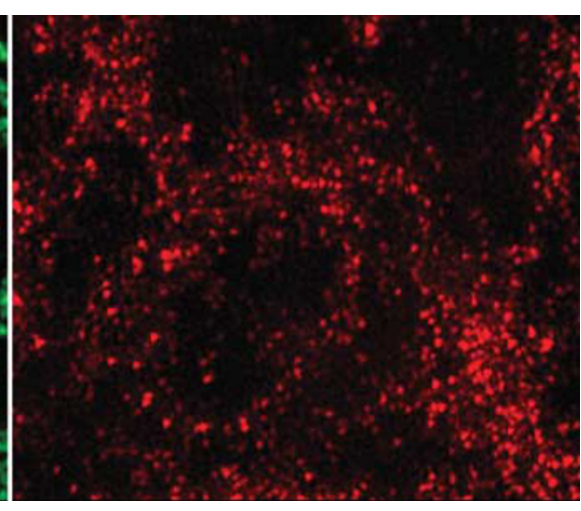

CED-9

b

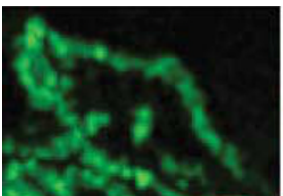

ATPsynthase

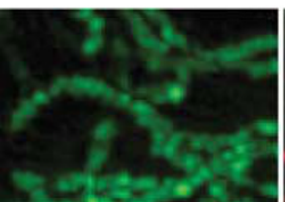

ATPsynthase

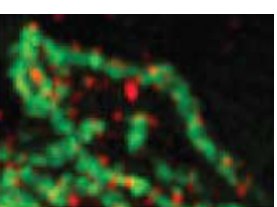

MERGE

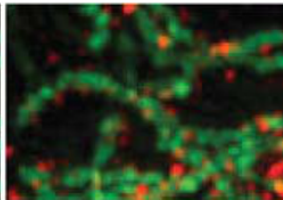

MERGE

C

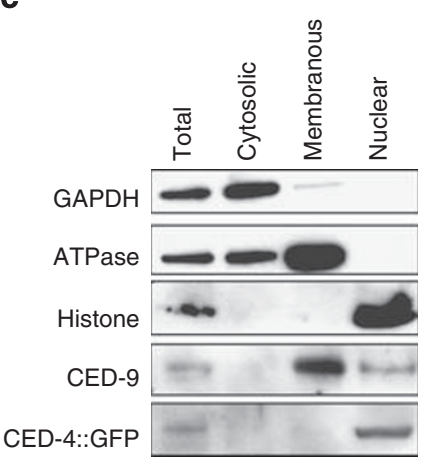

Figure 4 (a) Punctate CED-9 staining is associated with mitochondria. (b) A blow up of mitochondrial branches (indicated by arrows) is shown in the lower right panels. Scale bar: $4 \mu \mathrm{m}$. A 3D scan of the picture is shown in Supplementary Movie 6. (c) Subcellular fractionation. Mixed-stage C. elegans extracts were generated and fractionated as described in Materials and Methods. Enrichment of subcellular compartments was confirmed using antibodies against the indicated marker proteins

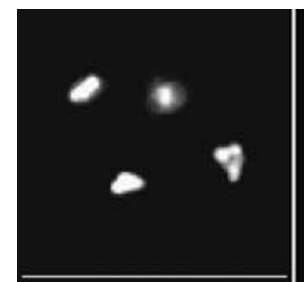

DAPI

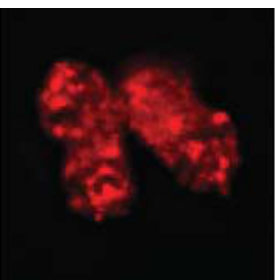

CED-4

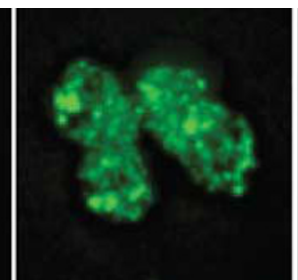

CED-9

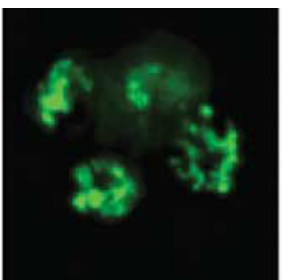

Mitochondria

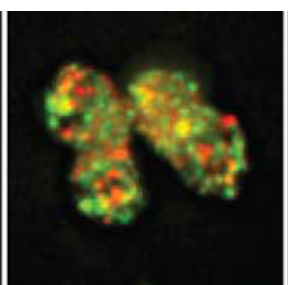

CED-4, CED-9

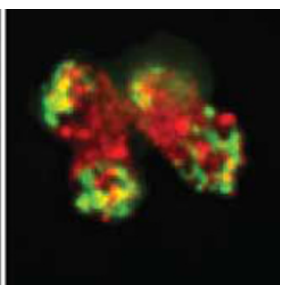

CED-4, Mitochondria

Figure 5 CED-4 and CED-9 staining overlaps in secondary spermatocytes. The CED-4 (10147.1) antibody was used ${ }^{18}$

corpses (5 and 6), chromatin had started to condense and CED-4 staining had disappeared (Figures 1b and 6; Supplementary Movies 8 and 9). Thus, CED-4 hyperaccumulation seems to be a consequence of corpse degradation and only occurs in very few late-stage apoptotic cells. To more precisely determine where CED-4 localizes before and after ionizing radiation, we used OMX microscopy to observe CED4 and nuclear pore complexes by immunostaining, and found 
Non-IR

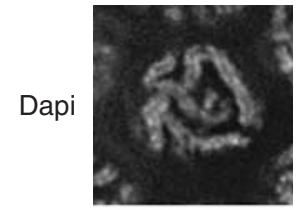

CED-4
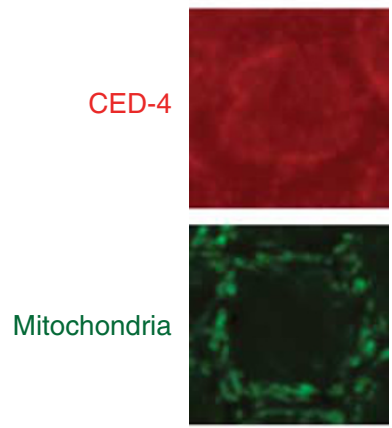

CED-4,

Mitochondria

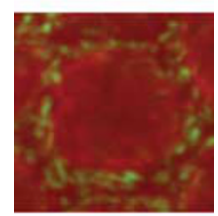

SIR-2.1

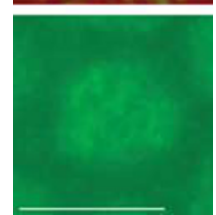

Early corpse
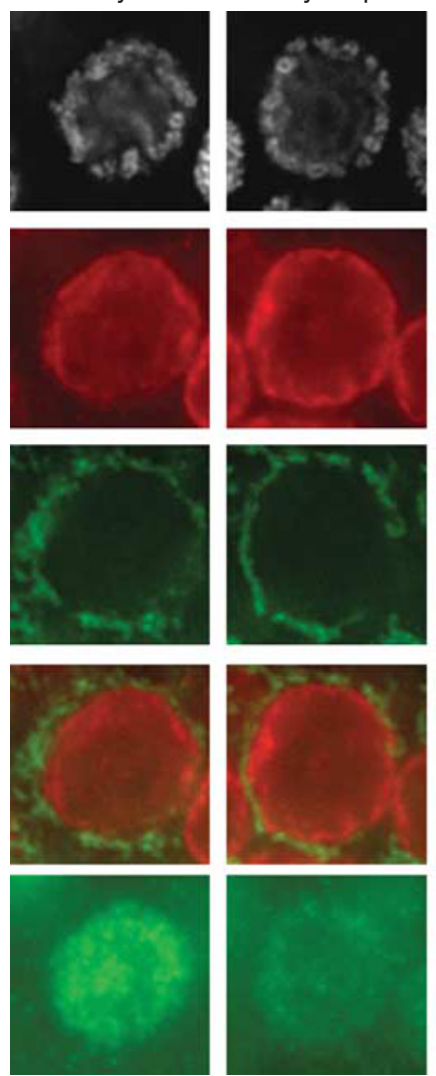

1

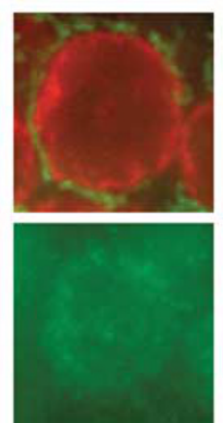

2

$R$
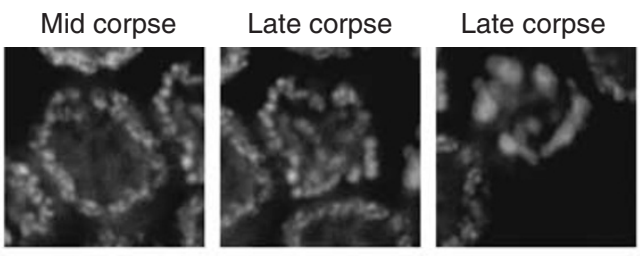

Very late corpse
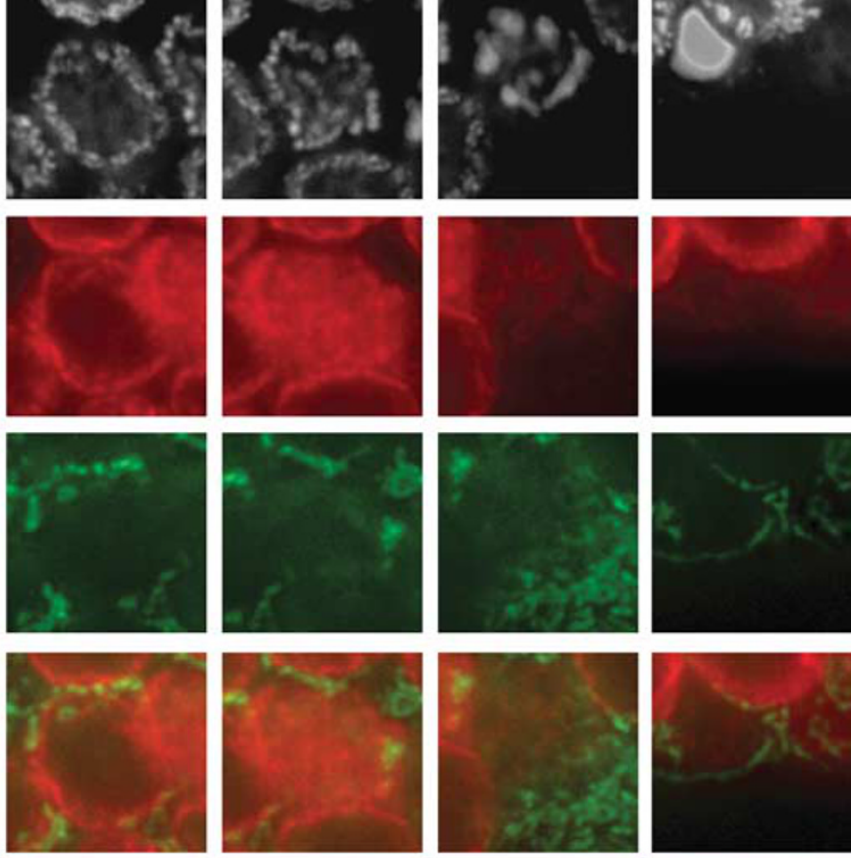

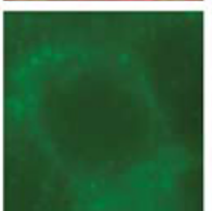

3

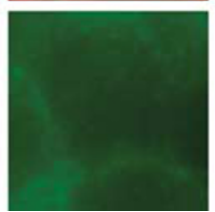

4

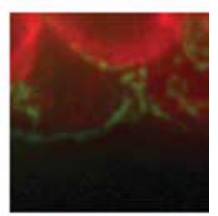

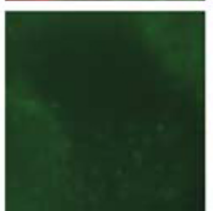

5

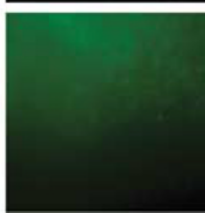

6

Figure 6 Various stages of germ cell apoptosis. Cells/corpses shown are indicated in Figure 1b. Scale bar: $6 \mu \mathrm{m}$

N2

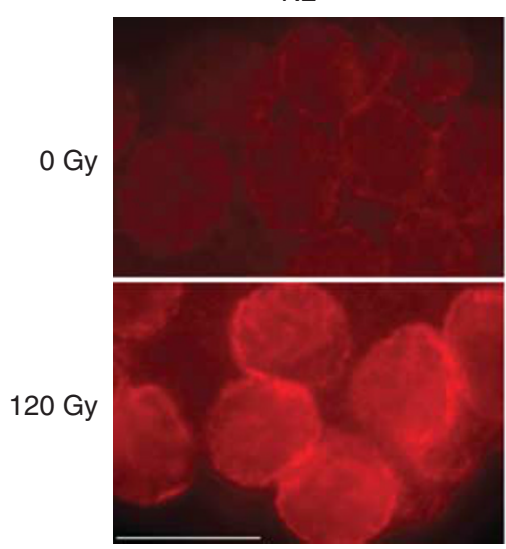

egl-1

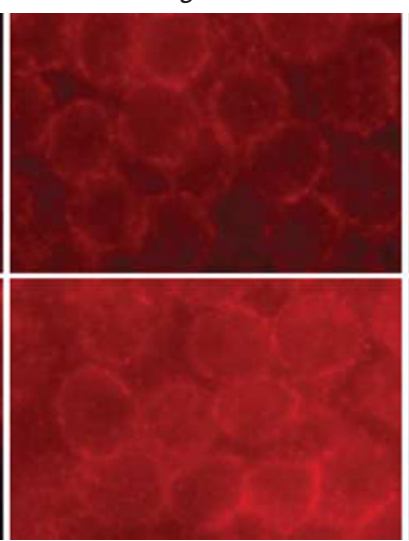

ced-9 (gf)

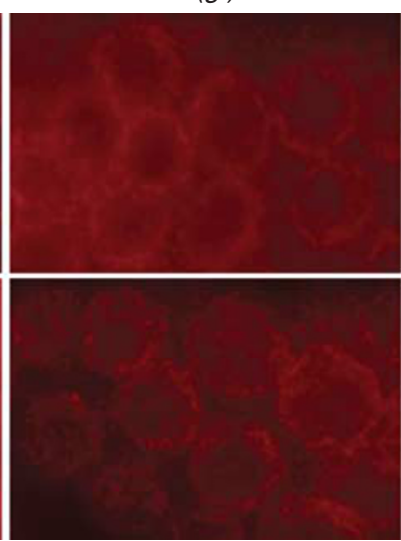

ced-9 (If), ced-3

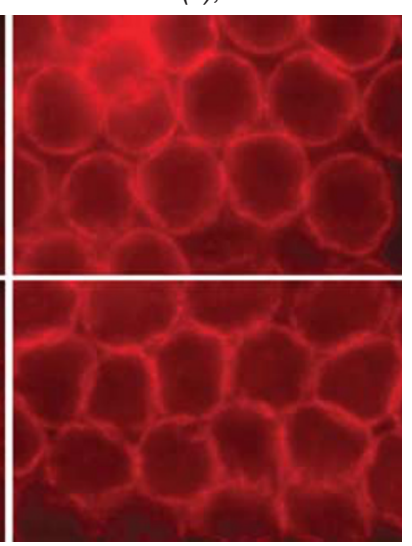

Figure 7 CED-4 perinuclear accumulation upon IR is absent in egl-1 loss-of-function and ced-9 gain-of-function mutants. The CED-4 (9104.1) antibody was used. Scale bar: $10 \mu \mathrm{m}$

that CED-4 is located outside of the nucleus before and after irradiation (Figure 1c). Unfortunately, OMX microscopy does not permit quantification of the CED-4 perinuclear accumulation in irradiated germ cells. We next wished to establish if the core apoptosis pathway is needed for CED-4 perinuclear accumulation. We analyzed an egl-1 loss-of-function mutant as well as a ced-9 gain-of-function mutant, both of which have dramatically reduced levels of DNA damage-induced germ cell apoptosis. We found that CED-4 fails to accumulate at the nuclear periphery in response to IR in an egl-1(If) as well as in a ced-9(gf) background (for representative pictures, see Figure 7$)$. In contrast to this, CED-4 strongly accumulates at the nuclear periphery in ced-9(If); ced-3(If) double mutants, where apoptosis induction is triggered by the loss of CED-9, whereas apoptosis execution is blocked by the absence of the CED-3 caspase. Thus, we confirm previous data obtained 
from observing embryos suggesting that the loss of CED-9 leads to CED-4 perinuclear accumulation, ${ }^{6}$ but our combined data suggest that at least during germ cell apoptosis in wildtype animals, this accumulation is not sufficient for apoptosis induction.

\section{Discussion}

In summary, we provide evidence that apoptosis regulation mediated by CED-4 and CED-9 is unlikely to be solely explained by a direct interaction between these two proteins. Although we found colocalization of CED-4 and CED-9 at mitochondria in spermatocytes and were thereby able to confirm that the reported in vitro interaction between CED-4 and CED-9 indeed appears possible in vivo, our data clearly show that in the majority of tissues CED-4 and CED-9 do not overlap. We cannot rule out the possibility that a small undetectable fraction of CED-4 directly interacts with CED-9 in mitochondria. Alternatively, a small fraction of CED-9 might be in the nucleus where it could colocalize with CED-4. We think that the CED-9 background staining in the nuclear fraction of the cell fractionation experiment is likely because of imperfect fractionation, but we cannot rule out that some CED9 not detectable by cytology might be associated with the nucleus. Nevertheless, we clearly show that the vast majority of CED-4 is not associated with CED-9, a localization pattern that according to the current model should result in active CED-4 and initiation of apoptosis. It has been previously reported that an antiapoptotic isoform of CED-4, CED-4L, exists. $^{26}$ We were not able to differentiate between the canonical CED-4 (CED-4S) and CED-4L in western blots, likely because those two isoforms only differ by 22 amino acids present in CED-4L and missing in CED-4S. ${ }^{26}$ Given that we used polyclonal antibodies that were raised against the entire CED-4S protein, we think it is highly unlikely that our staining only detect the antiapoptotic CED-4 isoform. Our data on CED-4 localization are largely based on the $C$. elegans germ line system. Extruding germ lines generally allows for more efficient antibody penetration and staining when compared with embryos, where staining procedures require permeabilization of the egg shell. Nevertheless, the convergence of the CED-4 localization pattern that we observed by combined cytological and biochemical methods, as well as the imaging of CED-4::GFP in the germ line and during embryogenesis, indicates that the localization of CED-4 does not fundamentally differ between the germ line and during development.

Our notion that apoptosis induction might not be regulated by the direct interaction between CED-4 and CED-9 is in agreement with a recent report showing that a CED-9 mutant defective in CED-4 binding is still able to rescue the embryonic lethality associated with the excessive apoptosis in a ced-9 loss-of-function background. ${ }^{27}$ As in mammals, $C$. elegans apoptosis induction may require additional, as of yet unknown factors for the activation of CED-4. In mammals, cytochrome $c$ release from mitochondria is required for Apaf-1 activation. However, the missing activator of CED-4 is unlikely to be cytochrome $c$, as CED-4 lacks the WD40 domain needed for cytochrome $c$ binding. Furthermore, we did not observe mitochondrial release of cytochrome $c$ in response to apoptotic stimuli (data not shown). Analogous to mammalian systems, the release of $C$. elegans AIF and EndoG from mitochondria, as well as mitochondrial fragmentation, have been implicated in $C$. elegans apoptosis induction. However, only a weak apoptosis-defective phenotype is observed following depletion of AIF and EndoG. ${ }^{13,14}$ Nevertheless, CED-9 might have a role in regulating the release of mitochondrial proteins. The $\mathrm{Bcl}-2$ family of proteins is related to bacterial pore-forming proteins and $\mathrm{Bcl}-2$ has been shown to be able to form pores in synthetic membranes. ${ }^{15,28-30}$ Furthermore, CED-9 is able to insert into artificial membranes causing membrane permeabilization and the fusion of membrane vesicles. ${ }^{15}$ These findings are in line with recent reports showing that CED-9 overexpression leads to the excessive fusion of mitochondrial membranes in not only mammalian tissue culture systems, but also in various C. elegans tissues. ${ }^{16,17}$ In summary, our results are not consistent with the widely held notion that the decision between $C$. elegans cell survival and cell death is mediated by a direct interaction of the Apaf-1-like molecule CED-4 and Bcl-2-like CED-9 in vivo. It will be interesting to explore how CED-9 controls CED-4 activation in vivo and if this relates to changes in mitochondrial permeability or dynamics.

\section{Materials and Methods}

C. elegans strains. C. elegans strains were maintained at $20^{\circ} \mathrm{C}$ on NGM plates and were fed with E. coli (OP50 strain) as described previously. ${ }^{31}$ MT2547 ced-4 (n1162)III; MT7386 ced-9 (n2812)III, ced-3(n717)IV; MT4771 ced-9(n1950) III; TG1768 egl-1(n3082)V; TG1765 ced-3(n717) IV; TG1766 ced-4(n1162) unc-119(ed3)III; TG1767 gt1767 Ex[pced-4:.:ced-4::gfp::ced-4 3'UTR, unc-119 $(+)] c e d-4(n 1162)$ unc-119(ed3).

Antibodies. To generate the 9104.1 rabbit anti-CED- 4 antiserum, $6 \times$ Histagged CED-4 (pGA333) was used for immunization, and MBP (maltose-binding protein)-tagged CED-4 (pGA334) was used to purify the antibody as described by Greiss et al. ${ }^{18}$ The 9103.1 rabbit CED-4 antibody and the 10147.1 goat CED-4 antibody are described by Greiss et al., ${ }^{18}$ and anti-CED-9 antibodies are described by Rolland et al. ${ }^{17}$

Western blotting. Worms were lysed using a mini beadbeater (BioSpec Products, Bartlesville, VA, USA) $(3 \times 20 \mathrm{~s})$ set to maximum, using $0.7 \mathrm{~mm}$ Zirconia Beads (BioSpec Products) in $150 \mu$ l of $7 \mathrm{M}$ urea lysis buffer containing $0.1 \mathrm{M} \mathrm{DTT}$, $0.05 \%$ Triton X-100, $25 \mathrm{mM} \mathrm{NaCl}$ and $20 \mathrm{mM}$ HEPES/KOH, $\mathrm{pH}$ 7.5. The protein lysate was incubated on ice for $30 \mathrm{~min}$ followed by $20 \mathrm{~min}$ of centrifugation at 13000 r.p.m. in an Eppendorf tabletop centrifuge (Hamburg, Germany) at $4^{\circ} \mathrm{C}$. Total protein concentration was measured by Bradford assay (Bio-Rad, Hercules, CA, USA). Lysate equivalent to $10 \mu \mathrm{g}$ of protein was separated by SDS-PAGE electrophoresis using 4-12\% gradient gels (Invitrogen, Carlsbad, CA, USA). Proteins were transferred to nitrocellulose membrane using the Invitrogen iBlot system, followed by blocking with PBST (PBS supplemented with $0.1 \%$ Tween-20) containing $5 \%$ milk powder for 30 min at RT. Probing with anti-CED-9 antibody $(1: 1000)$ was performed overnight at $4^{\circ} \mathrm{C}$. Washing was done three times at $\mathrm{RT}$ for 10 min each with ice-cold washing buffer (PBS containing $0.1 \%$ Tween-20). HRPconjugated donkey anti-rabbit antibodies (Jackson Immunochemicals, West Grove, PA, USA) were used as secondary antibodies in a dilution of $1: 10000$. Incubation was at RT.

\section{Immunostaining and microscopy}

Germ line staining for Deltavision microscopy. Immunostaining of germ lines and imaging using Deltavision microscopy was done as described by Greiss et al.. ${ }^{18}$ using the following antibodies and dilutions: rabbit anti-CED-4 (9103.1) $1: 100$, rabbit anti-CED-4 (9104.1) $1: 200$, rabbit anti-CED-9 (CED-9.1) $1: 150$ and goat anti-SIR-2.1 (126.1) 1:100. Antibodies used for mitochondrial staining are as described by Greiss et al., ${ }^{18}$ using a $1: 200$ dilution, as follows: anti-ATP synthase subunit- $\alpha$ (Mitosciences, Eugene, OR, USA; MS507), anti-cytochrome $c$ (MSA06); complex IV subunit 1 (MS404) and Pyruvate Dehydrogenase (MSP07) ATP 
synthase subunit- $\beta$ (MS503). The ER was stained using anti-HDEL SC-53472 (Santa Cruz, Santa Cruz, CA, USA) (1:100). The following secondary antibodies were used for detection (all from Molecular Probes, Eugene, OR, USA): Alexa 488-labeled donkey anti-goat $1: 200$, Alexa 568-labeled donkey anti-rabbit 1:500, Alexa 647-labeled donkey anti-mouse 1:200, Alexa 488-labeled goat antimouse IgG2a antibody $1: 200$, Alexa 568-labeled goat anti-mouse IgG1 antibody $1: 200$ and Alexa 647-labeled goat anti-mouse lgG2b antibody 1:200.

Embryo. Sample preparation was done on poly-lysine $(0.1 \% \mathrm{w} / \mathrm{v}$, Sigma, St. Louis, MO, USA)-coated superfrost Plus slides (VWR International, West Chester, PA, USA) and fixation was done as described for the germ line with the following modifications: ${ }^{18}$ post fixation was done at RT. Pre-blocking with Image IT FX signal enhancer (Invitrogen) was done for $15 \mathrm{~min}$. For the staining shown in Figure 2, the CED-4::GFP fusion was detected by the 'GFP booster, Chromotex' anti-GFP monovalent antibody that is directly coupled to ATTO 488 fluorescent dye, and which was incubated $(1: 200)$ with the secondary antibodies in this straining. Antibodies were used at the same dilution as for the germ line staining.

Staining for SI-OMX microscope. Embryos were prepared and fixed as described above ${ }^{18}$ except for the permeabilization, which was done by four 10-min incubations at RT, and preblocking with Image IT FX signal enhancer (Invitrogen), which was done for $10 \mathrm{~min}$ at RT. Primary antibodies and their dilutions were as follows: IgG2b anti-ATP synthase subunit- $\alpha$ (MS507) 1:150, IgG2a anticytochrome $c$ (MSA06) 1:150 and CED-9.1 antibody 1: 100. Secondary antibodies were as follows: Alexa 568-labeled donkey anti-rabbit (Molecular Probes) 1:400, Alexa 488-labeled goat anti-mouse IgG2a antibody (Molecular Probes) 1:150, Alexa 488-labeled goat anti-mouse IgG2b antibody (Molecular Probes) 1:150 and DyLight 549 labeled Goat anti-mouse IgG2b (Jackson Immunochemicals) 1:100. Germ lines were fixed and stained as described above, except that germ lines were ruptured before fixation. Mouse monoclonal antibody MAb414 (1:150, Covance Research Products, Princeton, NJ, USA) was used to stain the nuclear pore complex.

Image analysis and 3D reconstruction. Images were analyzed using softWoRx Suite and softWoRx Explorer software (AppliedPrecision, Issaquah, WA, USA). SI-OMX 3D reconstructions and subtraction of noise were performed using Imaris software (Bitplan AG, Scientific Software, Zurich, Switzerland).

Generation of transgenic worms. The sequence of the ced-4::gfp fusion plasmid (pGA402) containing ced-4 promoter, ced-4 genomic sequence followed by GFP inserted right before the stop codon and the ced-4 $3^{\prime}$-UTR can be obtained upon request. The ced-4 promoter and ced-4 genomic sequences were amplified using the following primers:

GA2194: [5'-TGCAGGCGCGCCCATGCTTTCGTGGTGACATTG-3'] and

GA2199: [5'-AAAGCGGCCGCCACAGCATGCAAAATTTTTGAG-3'].

To clone the $3^{\prime}$-UTR,

GA2223a: [5'-TCGAGGCCGGCCTAAAACCTATCGTGTACAATATTG-3'] and GA2224: [5'-TCGAGGGCCCGTTCTTGCATTCGATCTTCTCAATTTG-3'] were used. To create transgenic lines, $5 \mu \mathrm{g}$ of pGA402 plasmid was used to transform TG1766 ced-4(n1162); unc-119(ed3) worms by biolistic bombardment using a PDS-100/He particle delivery system (Bio-Rad). ${ }^{32}$

\section{Subcellular fractionation}

Lysis of worms and release of cytoplasmic content. Worm pellet $(200 \mu \mathrm{l}$; mixed stage) was used. Worms were washed $3 \times$ in M9 buffer and collected by sedimentation. Worms were resuspended in $0.2 \mathrm{ml}$ ice-cold extraction buffer CE1 supplemented with protease inhibitor (Qiagen Qproteome Cell Compartment Kit, Qiagen, Hamburg, Germany). To disrupt the cuticles and to release the cells and cytoplasmic content, 100-150 $\mu \mathrm{l} 0.7 \mathrm{~mm}$ Zirconia Beads (BioSpec Products) were added to the vial, followed by bead beating (Mini-Beadbeater-8, BioSpec Products) at max speed for three times each $5 \mathrm{~s}$. Approximately $70 \%$ lysis was confirmed by microscopic observation, scoring for released nuclei. To avoid disrupting subcellular compartments, worms were not frozen before lysis. To further lyse the sample and to clear the lysate from non-lysed worms and worm cuticles, the sample was passed through cell-lysate homogenizer tubes (Qiashredder, Qiagen) and centrifuged for $2 \mathrm{~min}$ at $100 \times$ r.c.f. (Eppendorf cooling benchtop centrifuge). The lysate was then transferred to new homogenizer tubes and spun for $30 \mathrm{~s}$ at $100 \times$ r.c.f. and the procedure was repeated once more. After a final spin for $10 \mathrm{~s}$, the lysate was free from non-lysed worms and their cuticles. Subsequently, subcellular fractionation was performed according to the manufacturer's instructions, but using only $20 \%$ of the recommended volumes. After fractionation, the samples were incubated with 4 times their volume of acetone at $-20^{\circ} \mathrm{C}$ for $3 \mathrm{~h}$, followed by centrifugation at 13200 r.p.m. for $30 \mathrm{~min}$ at $4^{\circ} \mathrm{C}$. The supernatant was removed and the pellet was air dried. The pellet was resuspended in $100 \mu \mathrm{l}$ of $2 \%$ SDS and heated at $65^{\circ} \mathrm{C}$ for $30 \mathrm{~min}$ with occasional vortexing. Protein concentration of all the fractions was measured using the Pierce BCA protein concentration assay (Rockford, IL, USA) following the manufacturer's instructions. For western blotting, $5 \mu \mathrm{g}$ of each sample was used. The antibodies used were: anti-histone H2A antibody (Abcam, Cambridge, UK; AB13923) 1:1000; anti-ATP synthase subunit $\alpha$ antibody (Mitosciences) 1:2500; anti-GAPDH antibody (Sigma; G8795) 1:10000; antiGFP antibody (Cell Signaling Technology, Beverly, MA, USA; 2956) 1:1000 and anti-CED-9 antibody $1: 2000$.

\section{Conflict of Interest}

The authors declare no conflict of interest.

Acknowledgements. This research was funded by a CR-UK CDA (C11852/ A4500) award and by a Wellcome Trust Senior Research fellowship (0909444/Z/09/Z) to AG. EP was supported by a CRUK PhD studentship (C11852/A8052) and SG was supported by a Wellcome Trust Project grant (081923/Z/07/Z) to AG. We are grateful to Barbara Conradt for sharing CED-9 antibodies and to Ashley Craig and Ulrike Gartner for proofreading. We also thank Magali Billet for helping with the CED-4:GFP construct, Pablo Ibanez-Cruceyra for assisting with the generation of transgenic worms and Mark Larance for help relating to establishing subcellular fractionation. We are also thankful to The Dundee CLS imaging facility and particularly wish to thank Sam Swift and Emma King for their technical support. SULSA (Scottish Universities Life Science Alliance) supported the OMX microscope.

1. Ellis HM, Horvitz HR. Genetic control of programmed cell death in the nematode C. elegans. Cell 1986; 44: 817-829.

2. Conradt $B$, Horvitz HR. The $C$. elegans protein EGL-1 is required for programmed cell death and interacts with the Bcl-2-like protein CED-9. Cell 1998; 93: 519-529.

3. Hengartner MO, Ellis RE, Horvitz HR. Caenorhabditis elegans gene ced-9 protects cells from programmed cell death. Nature 1992; 356: 494-499.

4. del Peso L, Gonzalez VM, Inohara N, Ellis RE, Nunez G. Disruption of the CED-9.CED-4 complex by EGL-1 is a critical step for programmed cell death in Caenorhabditis elegans. J Biol Chem 2000; 275: 27205-27211.

5. Liu X, Kim CN, Yang J, Jemmerson R, Wang X. Induction of apoptotic program in cell-free extracts: requirement for dATP and cytochrome c. Cell 1996; 86: 147-157.

6. Chen F, Hersh BM, Conradt B, Zhou Z, Riemer D, Gruenbaum Y et al. Translocation of C. elegans CED-4 to nuclear membranes during programmed cell death. Science 2000; 287: 1485-1489.

7. Yang X, Chang HY, Baltimore D. Essential role of CED-4 oligomerization in CED-3 activation and apoptosis. Science 1998; 281: 1355-1357.

8. Yan N, Chai J, Lee ES, Gu L, Liu Q, He J et al. Structure of the CED-4-CED-9 complex provides insights into programmed cell death in Caenorhabditis elegans. Nature 2005; 437: 831-837.

9. Qi S, Pang Y, Hu Q, Liu Q, Li H, Zhou Y et al. Crystal structure of the Caenorhabditis elegans apoptosome reveals an octameric assembly of CED-4. Cell 2010; 141: 446-457.

10. Spector MS, Desnoyers S, Hoeppner DJ, Hengartner MO. Interaction between the C. elegans cell-death regulators CED-9 and CED-4. Nature 1997; 385: 653-656.

11. Chinnaiyan AM, O'Rourke K, Lane BR, Dixit VM. Interaction of CED-4 with CED-3 and CED-9: a molecular framework for cell death. Science 1997; 275: 1122-1126.

12. Yan N, Xu Y, Shi Y. 2:1 Stoichiometry of the CED-4-CED-9 complex and the tetrameric CED-4: insights into the regulation of CED-3 activation. Cell Cycle 2006; 5: 31-34.

13. Wang X, Yang C, Chai J, Shi Y, Xue D. Mechanisms of AlF-mediated apoptotic DNA degradation in Caenorhabditis elegans. Science 2002; 298: 1587-1592.

14. Parrish J, Li L, Klotz K, Ledwich D, Wang X, Xue D. Mitochondrial endonuclease G is important for apoptosis in C. elegans. Nature 2001; 412: 90-94.

15. Frederick JT, Zuckerman JE, Wells RC, Hill RB. The $C$. elegans b-cell lymphoma 2 (bcl-2) homolog cell death abnormal 9 (ced-9) associates with and remodels lipid membranes. Protein Sci 2011; 20: 62-74.

16. Delivani P, Adrain C, Taylor RC, Duriez PJ, Martin SJ. Role for CED-9 and Egl-1 as regulators of mitochondrial fission and fusion dynamics. Mol Cell 2006; 21: 761-773.

17. Rolland SG, Lu Y, David CN, Conradt B. The BCL-2-like protein CED-9 of C. elegans promotes FZO-1/Mfn1,2- and EAT-3/Opa1-dependent mitochondrial fusion. J Cell Biol 2009; 186: 525-540

18. Greiss S, Hall J, Ahmed S, Gartner A. C. elegans SIR-2.1 translocation is linked to a proapoptotic pathway parallel to cep-1/p53 during DNA damage-induced apoptosis. Genes Dev 2008; 22: 2831-2842. 
19. Schermelleh L, Carlton PM, Haase S, Shao L, Winoto L, Kner $P$ et al. Subdiffraction multicolor imaging of the nuclear periphery with $3 \mathrm{D}$ structured illumination microscopy. Science 2008; 320: 1332-1336.

20. Hockenbery D, Nunez G, Milliman C, Schreiber RD, Korsmeyer SJ. Bcl-2 is an inner mitochondrial membrane protein that blocks programmed cell death. Nature 1990; 348 334-336.

21. Riparbelli MG, Callaini G, Tripodi SA, Cintorino M, Tosi P, Dallai R. Localization of the Bcl-2 protein to the outer mitochondrial membrane by electron microscopy. Exp Cell Res 1995 221: 363-369.

22. Jaramillo-Lambert A, Harigaya $Y$, Vitt J, Villeneuve A, Engebrecht J. Meiotic errors activate checkpoints that improve gamete quality without triggering apoptosis in male germ cells Curr Biol 2010; 20: 2078-2089.

23. Gartner A, Milstein S, Ahmed S, Hodgkin J, Hengartner MO. A conserved checkpoint pathway mediates DNA damage-induced apoptosis and cell cycle arrest in $C$. elegans. Mol Cell 2000; 5: 435-443.

24. Deng $X$, Yin X, Allan R, Lu DD, Maurer CW, Haimovitz-Friedman A et al. Ceramide biogenesis is required for radiation-induced apoptosis in the germ line of C. elegans. Science 2008; 322: 110-115

25. Zermati Y, Mouhamad S, Stergiou L, Besse B, Galluzzi L, Boehrer S et al. Nonapoptotic role for Apaf-1 in the DNA damage checkpoint. Mol Cell 2007; 28: 624-637.
26. Shaham S, Horvitz HR. An alternatively spliced $C$. elegans ced-4 RNA encodes a novel cell death inhibitor. Cell 1996; 86: 201-208

27. Dreze M, Charloteaux B, Milstein S, Vidalain PO, Yildirim MA, Zhong $Q$ et al. 'Edgetic' perturbation of a C. elegans BCL2 ortholog. Nat Methods 2009; 6: 843-849.

28. Muchmore SW, Sattler M, Liang H, Meadows RP, Harlan JE, Yoon HS et al. X-ray and NMR structure of human Bcl-xL, an inhibitor of programmed cell death. Nature 1996; 381: 335-341.

29. Hengartner MO. The biochemistry of apoptosis. Nature 2000; 407: 770-776.

30. Desagher S, Martinou JC. Mitochondria as the central control point of apoptosis. Trends Cell Biol 2000; 10: 369-377.

31. Brenner S. The genetics of Caenorhabditis elegans. Genetics 1974; 77: 71-94.

32. Praitis V, Casey E, Collar D, Austin J. Creation of low-copy integrated transgenic lines in Caenorhabditis elegans. Genetics 2001; 157: 1217-1226.

This work is licensed under the Creative Commons Attribution-NonCommercial-Share Alike 3.0 Unported License. To view a copy of this license, visit http://creativecommons. org/licenses/by-nc-sa/3.0/

Supplementary Information accompanies the paper on Cell Death and Differentiation website (http://www.nature.com/cdd) 